\title{
Intracranial EEG and laser interstitial thermal therapy in MRI-negative insular and/or cingulate epilepsy: case series
}

\author{
Elakkat D. Gireesh, MD, ${ }^{1}$ Kihyeong Lee, MD, ${ }^{1}$ Holly Skinner, DO, Joohee Seo, MD, ${ }^{1}$ \\ Po-Ching Chen, PhD, ${ }^{1,4}$ Michael Westerveld, PhD, ${ }^{3}$ Richard D. Beegle, MD, ${ }^{5}$ \\ Eduardo Castillo, $\mathrm{PhD},{ }^{4}$ and James Baumgartner, $\mathrm{MD}^{2}$
}

${ }^{1}$ Epilepsy Center, Neuroscience Institute, AdventHealth; ${ }^{2}$ Department of Neurosurgery, Neuroscience Institute, AdventHealth; ${ }^{3}$ Department of Neuropsychology, Neuroscience Institute, AdventHealth; ${ }^{4} \mathrm{MEG}$ Center, Neuroscience Institute, AdventHealth; and ${ }^{5}$ Department of Radiology, AdventHealth, Orlando, Florida

OBJECTIVE The goal of this study was to assess the success rate and complications of stereo-electroencephalography (sEEG) and laser interstitial thermal therapy (LITT) in the treatment of nonlesional refractory epilepsy in cingulate and insular cortex.

METHODS The authors retrospectively analyzed the treatment response in 9 successive patients who underwent insular or cingulate LITT for nonlesional refractory epilepsy at their center between 2011 and 2019. Localization of seizures was based on inpatient video-EEG monitoring, neuropsychological testing, 3-T MRI, PET scan, magnetoencephalography scan, and/or ictal SPECT scan. Eight patients underwent sEEG, and 1 patient had implantation of both sEEG electrodes and subdural grids for localization of epileptogenic zones. LITT was performed in 5 insular cases (4 left and 1 right) and 3 cingulate cases (all left-sided). One patient also underwent both insular and cingulate LITT on the left side. All of the patients who underwent insular LITT as well as 2 of the 3 who underwent cingulate LITT were right-hand dominant. The patient who underwent insular plus cingulate LITT was also right-hand dominant.

RESULTS Following LITT, $67 \%$ of the patients were seizure free (Engel class I) at follow-up (mean 1.35 years, range 0.62.8 years). All patients responded favorably to treatment (Engel class I-III). Two patients developed small intracranial hemorrhages during the sEEG implantation that did not require surgical management. One patient developed a large intracranial hemorrhage during an insular LITT procedure that did require surgical management. That patient experienced aphasia, incoordination, and hemiparesis, which resolved with inpatient rehabilitation. No permanent neurological deficits were noted in any of the patients at last follow-up. Neuropsychological status was stable in this cohort before and after LITT.

CONCLUSIONS sEEG can be safely used to localize seizures originating from insular and cingulate cortex. LITT can successfully treat seizures arising from these deep-seated structures. The insula and cingulum should be evaluated more frequently for seizure onset zones.

https://thejns.org/doi/abs/10.3171/2020.7.JNS201912

KEYWORDS epilepsy surgery; insula; cingulate; laser ablation; stereo-EEG

A PPROXIMATELY $1 \%$ of the US population suffers from epilepsy, ${ }^{1}$ and $30 \%-35 \%$ of these patients have drug-resistant epilepsy (DRE). ${ }^{2}$ Patients with DRE can be treated with surgery designed to remove the seizure onset zone or to disrupt the epileptic network. Currently, long-term seizure control after epilepsy surgery ranges between $34 \%$ and $56 \%$ for extratemporal epilepsy. ${ }^{3-5}$ Failure to accurately identify the epileptogenic zone(s) and inadequate or inaccurate surgical interventions may contribute to epilepsy surgery failures.
When insular or cingulate cortex is suspected as the seizure onset zone, invasive monitoring of the structure would improve epilepsy management. ${ }^{6,7}$ Neither the insula nor the cingulate can be easily monitored using subdural electrode arrays. Attempts to monitor these structures by using combinations of subdural and depth electrodes have achieved inconsistent success. ${ }^{8}$ Recent advances in stereo-electroencephalography (sEEG) monitoring have helped to identify electrographic patterns from deep structures, including insular and cingulate cortex.,9-12

ABBREVIATIONS DRE = drug-resistant epilepsy; FSIQ = full-scale IQ; iMRI = intraoperative MRI; LITT = laser interstitial thermal therapy; MEG = magnetoencephalography; $\mathrm{OR}$ = operating room; RFTC = radiofrequency thermocoagulation; sEEG = stereo-electroencephalography; SMA = supplementary motor area.

SUBMITTED May 21, 2020. ACCEPTED July 13, 2020.

INCLUDE WHEN CITING Published online December 11, 2020; DOI: 10.3171/2020.7.JNS201912. 
Stereotactic robots with 3D navigational platforms allow accurate and efficient placement of sEEG electrodes into deep structures.

The fact that insula lies deep within the sylvian fissure and is adjacent to critical structures, including the internal capsule, makes it difficult to approach surgically. In the dominant hemisphere, the insula is covered by eloquent frontal, temporal, and parietal opercula. The cingulate gyrus is challenging to access surgically because of its location along the deep medial surface of the brain. Various minimally invasive approaches including stereotactic radiofrequency thermocoagulation (RFTC) $)^{11}$ and laser interstitial thermal therapy (LITT) have been used as a surgical strategy for managing epilepsy arising from the insula. RFTC performed to treat lesional insular epilepsy resulted in a seizure-free outcome in 53\% of patients, with a responder rate of $89 \%$ (described as Engel class I-III). Of the patients treated with insular RFTC, $42 \%$ experienced perioperative neurological deficits. In a recent study comparing LITT to open surgery for insular seizure foci, favorable outcomes were reported in $71 \%$ (Engel class I and II) in the LITT group and 75\% of those who underwent open surgery. ${ }^{13}$ We report our epilepsy center's experience in intracranial sEEG monitoring and subsequent LITT procedures in patients with MRI-negative epilepsy, localized to insular and/or cingulate regions.

\section{Methods}

\section{Inclusion Criteria}

Patients were selected using the following criteria: 1) DRE (documented, continued seizures despite trial of at least two antiepileptic drugs); 2) clinical suspicion of epilepsy originating from insular or cingulate regions; 3 ) negative MRI findings; 4) age 1-65 years; and 5) epilepsy surgery between January 1, 2011, and January 30, 2019. The institutional review board at AdventHealth Orlando approved this retrospective chart review.

\section{Presurgical Workup}

Presurgical evaluation included a scalp video-EEG evaluation; MRI of brain including diffusion tensor imaging sequences; attempted ictal SPECT, PET, and magnetoencephalography (MEG); and neuropsychological evaluation to localize the epileptogenic zones. MEG included sensorimotor and language mapping. All cases were discussed at our multidisciplinary epilepsy surgery conference, and treatments were planned based on conference consensus. Wada testing and functional MRI were done if deemed necessary based on conference decision.

\section{Electrode Placement and Intracranial EEG Monitoring}

For sEEG electrode placement, planning was performed with ROSA planning software, using contrastenhanced T1-weighted MR images to identify and avoid vascular structures. Eight patients had intracranial monitoring with sEEG electrodes. The remaining patient was evaluated using a hybrid of grid and sEEG electrodes, with depth electrodes placed into the insular regions.

Electrodes were placed in an operating room (OR) equipped with a Siemens Somatom Open intraoperative CT. For sEEG procedures, electrode arrays were implanted as follows: skull bone fiducial, 10- to 14-mm titanium screw-based (DePuy Synthes) sterile registration was performed using a Leksell frame (Elekta) and a ROSA robot (Zimmer Biomet). sEEG electrodes were placed stereotactically through twist drill trephinations and anchor bolts were inserted (electrodes and anchor bolts from PMT, Inc.) at planned entry sites along planned trajectories. For hybrid monitoring (sEEG plus subdural electrodes), sEEG electrodes were placed as described above; then the patient was taken out of the Leksell frame, and a craniotomy was performed over the fronto-temporo-parietal region. Intraoperative electrocorticography was performed, after placing subdural grid and strip electrodes and additional electrodes as needed. Eight patients underwent sEEG electrode implantation, and 1 patient had a hybrid electrode array.

Oblique trajectories were used to place insular sEEG electrodes. The oblique trajectory allows multiple-contact $^{6-8}$ coverage of insular gray matter with each electrode (Fig. 1). In cases in which intraoperative electrocorticography suggested possible opercular involvement, additional transverse sEEG electrodes were placed during surgery. The cingulate electrodes were placed transversely in the initial cases. Because the area sampled with individual sEEG contacts is small and our experience with transversely placed cingulate electrode monitoring was inconclusive, we chose to monitor the cingulate gyrus longitudinally in the latter cases (Fig. 1). With electrodes placed through the long axis of the cingulum, we could more accurately assess seizure onset, spread, and the extent of involvement.

After placing the electrodes, a postoperative CT scan was obtained to document electrode position and to determine the presence or absence of intracranial hemorrhage. Patients were admitted to the neuro-ICU throughout the period of intracranial EEG monitoring. After capturing the patient's typical seizures, the case was discussed in a multidisciplinary epilepsy conference. We used the same skull bone fiducials for both sEEG and LITT fiber navigation, and therefore doing both procedures in the same hospital visit eliminated the need for reimaging.

\section{LITT Surgery}

Patients were taken to an intraoperative MRI (iMRI) OR suite with a 3-T Siemens Vario iMR imager. The sEEG electrodes and anchor bolts were removed using a sterile technique. The patients were then placed in an iMRI head holder for 3-point fixation, and a ROSA robot was attached to the Mayfield head holder by using a Monteris connector. The skull bone fiducials were then registered to the ROSA navigation system by using a sterile registration probe. An entry point and target point were then chosen for each LITT trajectory by using the ROSA navigation software. Using the ROSA drill guide attachment and the Monteris drill guide and drill bit, a twist drill trephination $(4.5 \mathrm{~mm})$ was created along the desired trajectory at the entry point. A Monteris minibolt was then advanced along the trajectory through the trephination with a Monteris minibolt hand driver. The accuracies of the trajectory and target were 

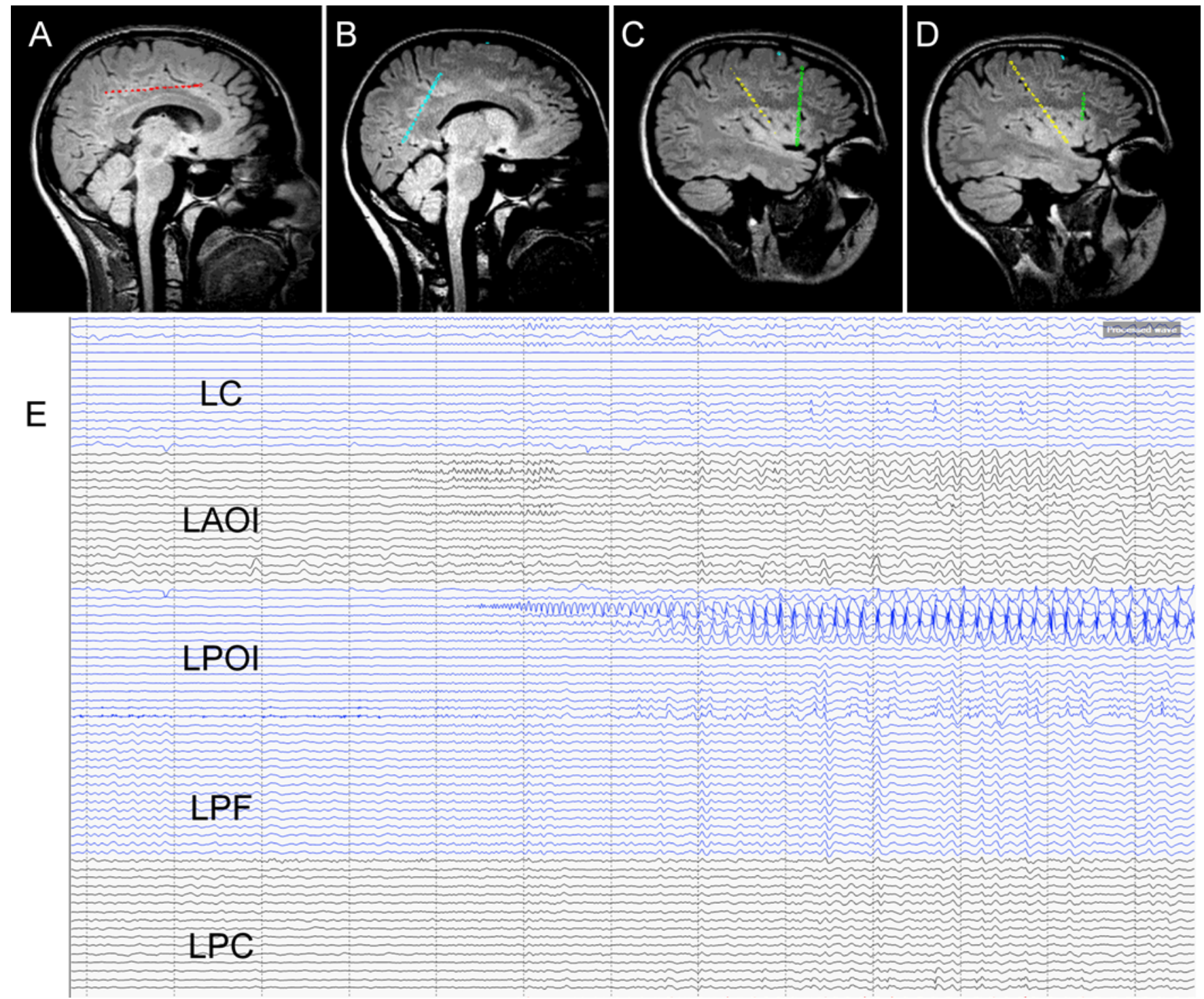

FIG. 1. Sagittal T2-weighted FLAIR MR images demonstrating insular and cingulate electrode position and the sEEG traces from the corresponding electrodes. A and B: Example of typical anterior (left cingulate) and posterior (left posterior cingulate) cingulate electrode placement. C and D: Example of typical insular anterior (left anterior oblique insular) and posterior (left posterior oblique insular) insular electrode placement. E: Seizures noted to be evolving near electrodes LAOI 2-4 and LPOI 3-5. LAOI = left anterior oblique insular; LC = left cingulate; LPC = left posterior cingulate; LPF = left posterior frontal; LPOI = left posterior oblique insular. Figure is available in color online only.

confirmed using Stryker neuronavigation registered to the skull bone fiducials. If multiple trajectories were deemed necessary, multiple minibolts were placed before ablation was initiated. Once all planned minibolts were in place, the ROSA robot was detached from the iMRI head holder and removed from the OR. A Monteris LITT robot was attached to the minibolt, and a Monteris 3.3-mm Side Fire navigable LITT fiber was advanced through the robot and minibolt to target depth. The iMRI imager was then moved into the OR and, under continuous MRI thermometry, laser ablation was performed sequentially from the deepest point along the trajectory to the most superficial point along the trajectory by using the Monteris software and workstation (Supplementary Fig. S1). The Monteris LITT robot was used to direct the laser fiber to achieve maximal ablation of epileptogenic tissue. This process was repeated until all minibolt-based trajectories had been ablated. An intraoperative T1-weighted MRI sequence with half-dose contrast was then obtained to estimate the extent of ablation achieved, and additional ablations were planned and performed if deemed necessary.

For cingulate ablations, the surgical approach for laser fiber placement was identical to that for the insular fiber placement. Because the cingulate gyrus is long and cylindrical, a 3.3-mm Monteris diffuse tip laser fiber was used to perform the ablation. Two cingulate ablations were completed through a single longitudinal trajectory. One cingulate LITT procedure was accomplished via 3 
TABLE 1. Clinical profiles in 9 patients with insular and/or cingulate epilepsy

\begin{tabular}{|c|c|c|c|c|c|c|c|c|c|}
\hline $\begin{array}{l}\text { Case } \\
\text { No. }\end{array}$ & Age & Handedness & Sex & $\begin{array}{l}\text { Age at } \\
\text { Seizure } \\
\text { Onset }\end{array}$ & $\begin{array}{l}\text { Seizure } \\
\text { Frequency }\end{array}$ & Medications & $\begin{array}{l}\text { Risk } \\
\text { Factors }\end{array}$ & $\begin{array}{l}\text { Neuro } \\
\text { Exam }\end{array}$ & $\begin{array}{l}\text { Prior } \\
\text { Surgery }\end{array}$ \\
\hline 1 & $7 \mathrm{yrs}$ & Rt & $\mathrm{F}$ & $6 \mathrm{yrs}$ & 10/day & OXC, VPA, CLP & NR & Cognitive deficits & None \\
\hline 2 & $46 \mathrm{yrs}$ & Rt & M & $27 \mathrm{yrs}$ & 1/night & CBZ, ZNS, GBP & Trauma & Cognitive deficits & None \\
\hline 3 & $19 \mathrm{yrs}$ & $\mathrm{Rt}$ & M & 3 wks & $5-7 /$ wk & PHT, CBZ, OXC & $\begin{array}{l}\text { Developmen- } \\
\text { tal delay }\end{array}$ & Cognitive deficits & Lt temporal lobectomy \\
\hline 4 & $22 \mathrm{yrs}$ & Rt & $\mathrm{F}$ & 16 yrs & $2-3 / w k$ & LTG, TPM, LEV, PMP & NR & Normal & None \\
\hline 5 & $20 \mathrm{yrs}$ & $\mathrm{Rt}$ & $\mathrm{F}$ & 4 yrs & $1-3 / w k$ & TPM, CBZ & $\begin{array}{l}\text { Low Apgar } \\
\text { score at birth }\end{array}$ & Normal & None \\
\hline 6 & 9 yrs & Rt & $\mathrm{F}$ & $6 \mathrm{yrs}$ & 5-10/day & LEV, LTG, VPA & $\begin{array}{l}\text { Family history } \\
\text { of epilepsy }\end{array}$ & Normal & None \\
\hline 7 & $18 \mathrm{yrs}$ & $\mathrm{Rt}$ & M & $12 \mathrm{yrs}$ & $1-3 / \mathrm{mo}$ & LTG, OXC, PHT, LEV, CLB & NR & $\begin{array}{l}\text { Rt-sided } \\
\text { weakness }\end{array}$ & Lt frontoparietal topectomy \\
\hline 8 & $18 \mathrm{yrs}$ & $\mathrm{Lt}$ & $\mathrm{F}$ & 4 mos & $>5 / w k$ & $\begin{array}{l}\text { PB, TPM, LTG, VPA, } \\
\text { ZNS, OXC }\end{array}$ & Autism & Cognitive deficits & $\begin{array}{l}\text { Corpus callosotomy, It pari- } \\
\text { etal topectomy, It frontal MST }\end{array}$ \\
\hline 9 & 6 yrs & $\mathrm{Rt}$ & $\mathrm{F}$ & $5 \mathrm{yrs}$ & 10-20/night & TPM, OXC, CLP & NR & Cognitive deficits & None \\
\hline
\end{tabular}

$\mathrm{CBM}=$ clobazam; $\mathrm{CBZ}$ = carbamazepine; $\mathrm{CLP}=$ clonazepam; $\mathrm{GBP}=$ gabapentin; $\mathrm{LEV}=$ leviteracetam; $\mathrm{LTG}=$ lamotrigine; $\mathrm{MST}$ = multiple subpial transections; neuro exam = neurological examination; NR = none reported; $\mathrm{OXC}=$ oxcarbazepine; $\mathrm{PB}=$ phenobarbital; $\mathrm{PHT}=$ phenytoin; $\mathrm{PMP}=$ perampanel; $\mathrm{TPM}=$ topiramate; VPA = valproic acid; ZNS = zonisamide.

orthogonal trajectories, because the desired longitudinal approach was not possible due to limiting paramedian vascular anatomy.

\section{Results}

\section{Population Studied}

Age at seizure onset for these patients ranged from 3 weeks to 27 years (average 8.5 years). The average age at the time of surgery was 18.3 years (range 6-46 years). There were 6 females and 3 males in this cohort of patients. The frequency of the seizures varied from multiple seizures in a day to 1-3 seizures per month. The risk factors for epilepsy included trauma, developmental delay, autism, and family history of seizures. In the patient with a family history of seizures, epilepsy was reported in the maternal grandmother, aunt, uncle, and two cousins. No genetic abnormalities were identified based on wholeexome sequencing. All patients had been treated with at least three antiseizure medications and met criteria for DRE. Three patients underwent prior unsuccessful epilepsy surgeries. The detailed clinical profiles of this group of patients are listed in Table 1.

\section{Presurgical Workup}

The semiology of our insular epilepsy cases included activity arrest, staring, lip smacking, contralateral facial and arm twitching, head drop, contralateral dystonic posturing, tingling sensation in throat, and tingling sensation in the contralateral arm. One of the patients had predominantly nocturnal seizures. Cingular seizures were commonly associated with contralateral dystonic movements (noted in 2 of the 3 cases). One patient with cingulate epilepsy had hypermotor seizures.

Indications for implanting electrodes in the insula in- cluded semiology, lack of clear lateralization of the scalp EEG findings, and epileptiform discharges noted from insular regions in the MEG sequence. The ictal onset zones noted in the scalp EEG included frontal, temporal, midline, central, or posterior temporal/parietal regions. One of the insular cases had beta wave activity noted in the midline. The MEG findings indicated an insular epileptogenic zone in 3 of the 6 insular cases.

Indications for implanting cingulate electrodes included seizure semiology, including early motor symptoms and unclear lateralization of the ictal scalp EEG. No clear MEG findings could be established in the patients with cingulate onset epilepsy.

\section{sEEG Monitoring}

We selected only the patients undergoing insular LITT during the period of study. The total number of patients undergoing sEEG in our center during this period was 76. Also, 172 patients underwent grid implantation during the same period. Four of the 5 insular cases had sEEG monitoring done bilaterally. The case without bilateral insular monitoring was evaluated with subdural grids and depth electrodes. Two of the 3 cingulate cases had bilateral sEEG monitoring. The patient who underwent both insular and cingulate LITT had bilateral insular and left-sided cingulate sEEG monitoring. Representative sEEG recordings with corresponding electrode positions are shown in Fig. 1. The sEEG findings are noted in Table 2. The details of sEEG electrodes and subdural grid coverage can be found in Table 3.

\section{LITT Procedure}

For LITT, trajectories of the sEEG electrodes with active contacts were used. We used the Monteris minibolt 
TABLE 2. Presurgical workup and sEEG findings in 9 patients with insular and/or cingulate epilepsy

\begin{tabular}{|c|c|c|c|c|c|c|c|c|}
\hline $\begin{array}{l}\text { Case } \\
\text { No. }\end{array}$ & Semiology & $\begin{array}{l}\text { Semiology } \\
\text { Localization }\end{array}$ & $\begin{array}{l}\text { Interictal } \\
\text { EEG }\end{array}$ & Ictal EEG & PET & SPECT & MEG & sEEG Seizure Onset \\
\hline 1 & Nocturnal; head version to It & Rt frontal & $\begin{array}{l}\text { Beta waves } \\
\text { over rt frontal }\end{array}$ & $\begin{array}{l}\text { Beta waves, } \\
\text { midline }\end{array}$ & $\begin{array}{l}\text { Lt } \\
\text { insular }\end{array}$ & $\begin{array}{l}\text { Lt temporal, } \\
\text { It insular }\end{array}$ & $\begin{array}{l}\text { Lt frontal, } \\
\text { It insular }\end{array}$ & Lt anterior insular \\
\hline 2 & $\begin{array}{l}\text { During sleep, dystonic } \\
\text { posturing of } \mathrm{rt} \text { arm/leg }\end{array}$ & $\begin{array}{l}\text { Lt frontal, It pa- } \\
\text { rietal, It insular }\end{array}$ & NL & $\mathrm{NL}$ & NL & $\mathrm{NL}$ & $\begin{array}{l}\text { Lt insular, } \\
\text { It perisylvian }\end{array}$ & Lt insular, It cingulate \\
\hline 3 & $\begin{array}{l}\text { Staring, activity arrest, lip smack- } \\
\text { ing, rt facial \& arm twitching }\end{array}$ & $\begin{array}{l}\text { Lt temporal, It } \\
\text { insular }\end{array}$ & $\begin{array}{l}\text { Lt central, } \\
\text { It temporal }\end{array}$ & $\begin{array}{l}\text { Lt frontal, } \\
\text { It temporal }\end{array}$ & Bilat & Rt temporal & $\begin{array}{l}\text { Lt temporal, } \\
\text { It insular }\end{array}$ & Lt insular, It temporal \\
\hline 4 & $\begin{array}{l}\text { Hypermotor (rt }>\text { It dystonic } \\
\text { posturing) }\end{array}$ & $\begin{array}{l}\text { Lt temporal, It } \\
\text { frontal }\end{array}$ & Lt frontal & $\begin{array}{l}\text { Lt central, } \\
\text { It parietal }\end{array}$ & $\mathrm{NL}$ & $\mathrm{Lt}>\mathrm{rt}$ & Lt frontal & Rt frontal, rt insular \\
\hline 5 & $\begin{array}{l}\text { Activity arrest, partial awareness, } \\
\text { staring, rt-sided dystonic } \\
\text { posturing }\end{array}$ & $\mathrm{Lt}$ & $\begin{array}{l}\text { Bilat frontal } \\
\text { sharp wave }\end{array}$ & $\mathrm{NL}$ & $\mathrm{NL}$ & Lt & $\mathrm{NL}$ & Lt posterior insular \\
\hline 6 & $\begin{array}{l}\text { Tingling sensation in rt forearm, } \\
\text { stiffening of } \mathrm{rt} \text { arm }\end{array}$ & $\mathrm{Lt}$ & Lt posterior & Lt posterior & $\mathrm{NL}$ & $\mathrm{Lt}$ & $\mathrm{Lt}$ & Lt anterior insular \\
\hline 7 & $\begin{array}{l}\text { Rt head deviation, rt-sided } \\
\text { dystonia }\end{array}$ & Lt frontal & $\begin{array}{l}\text { Lt central, } \\
\text { It temporal }\end{array}$ & Lt central & $\mathrm{NL}$ & $\mathrm{Lt}>\mathrm{rt}$ & Lt frontal & Lt cingulate \\
\hline 8 & $\begin{array}{l}\text { Arousal, frightened, rt arm \& } \\
\text { leg stiffening, head to rt }\end{array}$ & Lt frontal & NL & $\mathrm{Lt}$ & $\mathrm{NL}$ & $\mathrm{NL}$ & $\begin{array}{c}\text { Not } \\
\text { available }\end{array}$ & $\begin{array}{l}\text { Lt midcingulate spreading } \\
\text { to It posterior insular }\end{array}$ \\
\hline 9 & Hypermotor, flailing, drooling & Frontal & Bilat & $\mathrm{Lt}$ & NL & NL & Rt insular & Lt midcingulate \\
\hline
\end{tabular}

$\mathrm{NL}=$ nonlocalizing.

and robot for ablation, with a directional laser fiber, typically starting at the lowest power setting. When planned trajectories did not accomplish adequate ablation (3 cases), we used additional trajectories to expand the extent of ablation. Five patients had insular LITT (right side in 1, left side in 4). Three patients underwent cingulate cortex LITT only, all on the left side. One of the patients had LITT on both left cingulate and insular regions. A typical example of the MRI changes seen immediately following insular LITT and subsequent MRI findings 8 months later is shown in Fig. 2. The average volume of LITT in insular cases was $13.2 \mathrm{~cm}^{3}$ (range $5.3-29.2 \mathrm{~cm}^{3}$ ), and it was 15.3 $\mathrm{cm}^{3}$ (range 2.4-39.4 $\mathrm{cm}^{3}$ ) in cingulate cases (Table 3).

\section{Complications \\ sEEG-Related Complications}

sEEG-related bleeding was noted in 2 of the 9 cases. These included 1 case (the patient in case 7) of 3-mm asymptomatic subdural hematoma and 1 case (the patient in case 2) of intraparenchymal bleed (29 $\mathrm{mm}$ in longest dimension). The intraparenchymal bleed was not related to the insular sEEG electrodes and was located subcortically in the left parietal lobe, resulting in mild right hemiparesis. No lasting neurocognitive deficits were noted in association with these hemorrhages. One of the patients with cingulate foci developed a CSF leak, which was de-

TABLE 3. sEEG coverage, LITT procedures, and surgical outcomes in 9 patients with insular and/or cingulate epilepsy

\begin{tabular}{|c|c|c|c|c|c|c|c|c|c|c|}
\hline \multirow{2}{*}{$\begin{array}{l}\text { Case } \\
\text { No. }\end{array}$} & \multirow{2}{*}{$\begin{array}{c}\text { Total No. of } \\
\text { Electrode Contacts }\end{array}$} & \multicolumn{2}{|c|}{ Insular Implantation } & \multicolumn{2}{|c|}{ Cingulate Implantation } & \multirow{2}{*}{$\begin{array}{c}\text { LITT } \\
\text { Location }\end{array}$} & \multirow{2}{*}{$\begin{array}{l}\text { No. of LITT } \\
\text { Trajectories }\end{array}$} & \multirow{2}{*}{$\begin{array}{c}\text { LITT } \\
\text { Vol }\left(\mathrm{cm}^{3}\right)\end{array}$} & \multirow{2}{*}{$\begin{array}{c}\text { Surgical } \\
\text { Outcomes } \\
\text { (Engel class) }\end{array}$} & \multirow{2}{*}{$\begin{array}{l}\text { Follow-Up } \\
\text { (mos) }\end{array}$} \\
\hline & & Side & No. of Contacts & Side & No. of Contacts & & & & & \\
\hline 1 & 190 & Bilat & 24 & Bilat & 18 & Lt insular & 3 & 11.9 & $\mathrm{IA}$ & 22.67 \\
\hline 2 & 190 & Bilat & 24 & $\mathrm{Lt}$ & 26 & $\begin{array}{l}\text { Lt insular, } \\
\text { It cingulate }\end{array}$ & 2 & $29.2,39.4$ & IB & 15.7 \\
\hline 3 & $\begin{array}{c}116 \text { subdural, } 16 \\
\text { depth (grid electrodes) }\end{array}$ & $\mathrm{Lt}$ & 6 & NA & NA & Lt insular & 3 & 11.5 & $\| \mathrm{A}$ & 16.07 \\
\hline 4 & 215 & Bilat & 18 & Bilat & 8 & Rt insular & 2 & 7.9 & IA & 34.27 \\
\hline 5 & 250 & Bilat & 32 & Bilat & 48 & Lt insular & 3 & 5.3 & IA & 12.33 \\
\hline 6 & 212 & Bilat & 28 & $\mathrm{Lt}$ & 16 & Lt insular & 2 & * & IA & 10.2 \\
\hline 7 & 98 & NA & NA & $\mathrm{Lt}$ & 24 & Lt cingulate & 1 & 11.6 & IIIA & 15.3 \\
\hline 8 & 168 & Bilat & 20 & Bilat & 15 & Lt cingulate & 3 & 8 & IIB & 13.8 \\
\hline 9 & 202 & Bilat & 24 & Bilat & 12 & Lt cingulate & 1 & 2.4 & IB & 19.5 \\
\hline
\end{tabular}

$\mathrm{NA}=$ not applicable.

${ }^{*}$ Not available due to hemorrhage. 

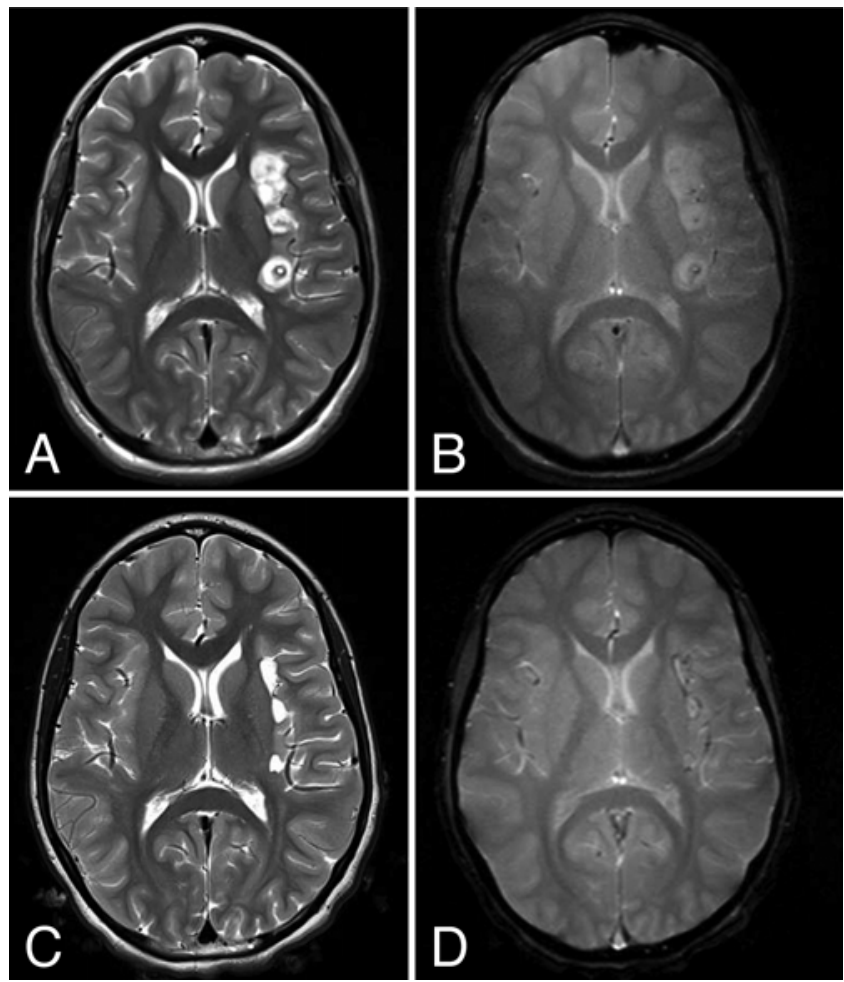

FIG. 2. Case 1. Chronological MRI changes after insular LITT. A and B: iMRI sequences obtained immediately after LITT. $C$ and D: MR images obtained 8 months after LITT. Panels $A$ and $C$ are axial T2 images. Panels $B$ and $D$ are axial gradient echo images.

tected after implantation and was repaired without incident.

\section{LITT-Associated Complications}

A supplementary motor area (SMA) syndrome was noted in the patient (case 2) who underwent both insular and cingulate LITT, leading to transient hemiparesis, hemineglect, and word-finding difficulty. These symptoms resolved with therapy. Two patients who underwent insular LITT (cases 4 and 5) experienced a mild transient SMA-like syndrome, which included expressive aphasia. One of the patients who underwent insular LITT (case 6) experienced a large intracerebral hemorrhage during the LITT that was detected by abrupt unexpected changes on thermometry. Due to these changes, LITT was stopped and MRI performed, revealing a large intracerebral hemorrhage. Immediately after the completion of the LITT procedure, the patient underwent an emergency large decompressive craniectomy with placement of a dural expansion graft and an intracranial pressure monitor. At that time, the brain was soft and pulsatile. Angiography was postponed out of concern that the intracerebral hemorrhage might distort the vasculature. Postoperatively, the patient was extubated and found to have a mixed aphasia and right-sided weakness. She remained awake and able to follow some commands with an intracranial pressure below $15 \mathrm{~mm} \mathrm{Hg}$ postoperatively. After inpatient rehabilitation, she underwent angiography (negative findings) and replacement of her bone flap (Supplementary Fig. S2). At the 6-month follow-up, she had very mild slowing in fine finger movements of her right hand. At the 1-year followup, her fine finger movements were symmetrical, and she walked and ran without difficulty. She regained baseline levels of expressive and receptive language skill.

\section{Seizure Outcomes}

Postoperative patient follow-up ranged from 10 months to 34 months (Table 3). Six of the 9 patients (67\%) achieved complete seizure freedom (Engel class I), and all patients had a favorable seizure response (Engel class I-III). Four of the 5 patients with insular epilepsy, and 1 of 3 patients with cingulate epilepsy were seizure free (Engel class I). The patient who underwent both insular and cingulate LITT also achieved seizure freedom. Two patients (cases 4 and 5) had partial seizure control (Engel class III) after initial LITT treatment. They later underwent additional surgical procedures (the patient in case 4 had left anterior cingulate topectomy and corpus callosotomy, and the patient in case 5 had a second LITT; Fig. 3) before achieving seizure freedom. No significant statistical difference was noted in the different parameters between the patients who had better versus relatively worse outcome (Supplementary Table S1).

\section{Neuropsychological Outcomes}

All patients underwent comprehensive neuropsychological evaluation (Supplementary Table S2). The full-scale IQ (FSIQ) score prior to surgery ranged from 64 to 108 (available only for 7 patients) and was 58-100 (5 patients) after surgery. No significant differences were noted when the pre-FSIQ and post-FSIQ scores were compared in the available pairs $(\mathrm{n}=5, \mathrm{p}=0.4)$. There were no definite trends in the ABAS (Adaptive Behavior Assessment System) scores before and after surgery.

\section{Discussion}

\section{Key Results}

Following intracranial monitoring and LITT of the insula and cingulum, 6 of 9 patients in our cohort became seizure free (Engel class I), 2 of 9 reached an Engel class II outcome, and 1 of 9 achieved an Engel class III outcome with MRI-negative epilepsy. Previous studies have reported the technique and safety of the sEEG electrode implantation for insular epilepsy. ${ }^{14}$ The patients in this group suffered from long-standing intractable epilepsy. Three of our 9 patients had undergone unsuccessful epilepsy surgery (Table 1) previously. In those patients, the initial workup and intracranial subdural electrode arrays placed during the initial phase 2 surgical treatment did not include insular or cingulate electrodes. All 3 patients experienced improved seizure control after sEEG and LITT targeting these structures. This experience is consistent with previous reports that clinical manifestations and the preliminary workups of this patient population can be misleading. ${ }^{15}$

Previous surgical series report variable degrees of seizure freedom, ranging from $56 \%{ }^{16}$ to $69 \%,{ }^{17}$ following insular resection. Most of the patients in these series had lesions in or near insular cortex. In a pediatric study comparing open resection versus LITT for insular epilepsy, le- 


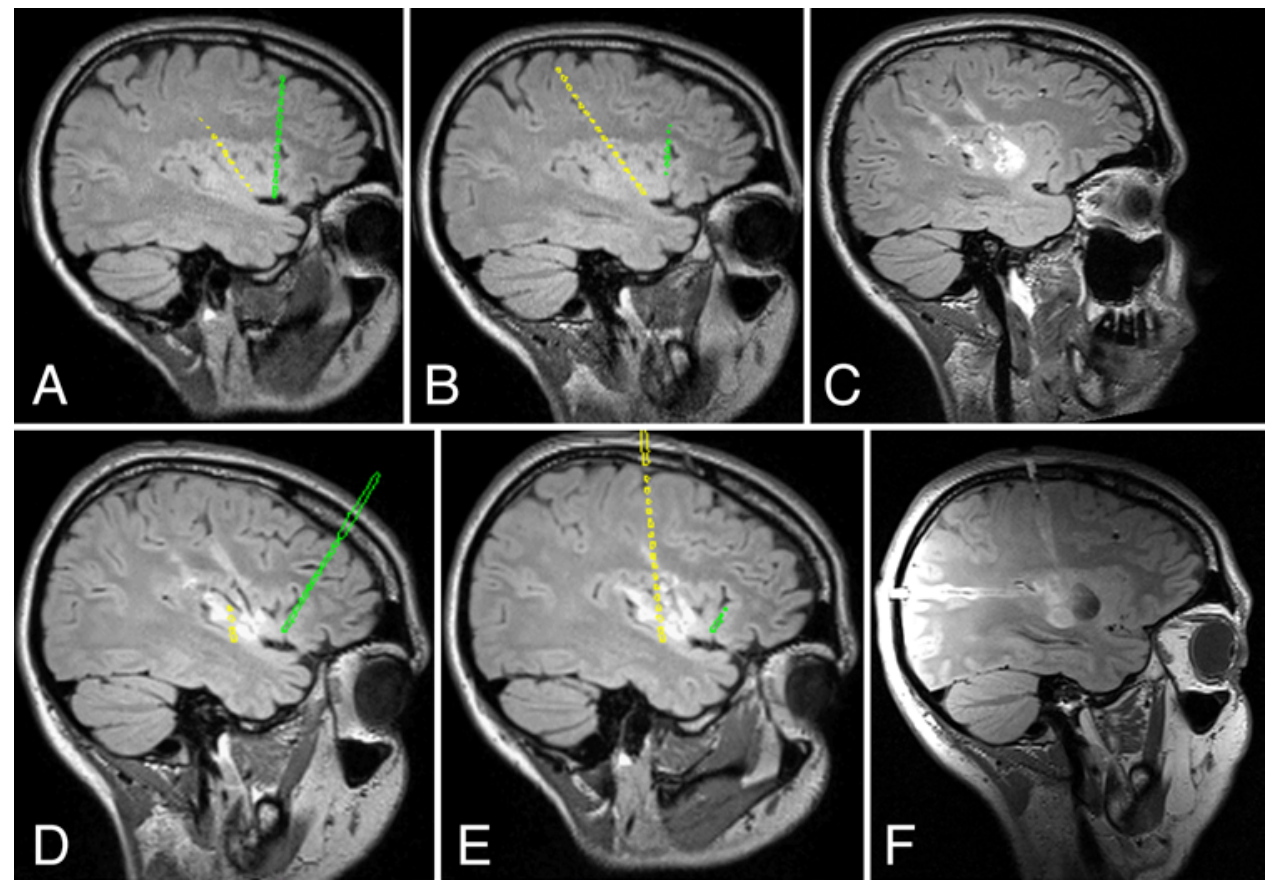

FIG. 3. Case 5. LITT of the left insula. A-C: Sagittal views showing the initial insular electrode implantation and the results of the first LITT. D-F: Sagittal views showing the second insular electrode implantation and the results of the second LITT. Panels A and $D$ illustrate left anterior insular electrode locations. Panels B and E illustrate left posterior insular electrode locations. Panels $C$ and $F$ are T2-weighted FLAIR images obtained after the second LITT. The sEEG recordings from the posterior insular electrode (yellow, panel E) demonstrated epileptogenic activity. Figure is available in color online only.

sions were identified on MR images in $60 \%$ of patients, and Engel class I outcome was reported in $43 \%$ of subjects with at least a 1-year follow-up. In that cohort, 3 of the 6 nonlesional cases achieved Engel class I outcome. In another recent study in which RFTC-based surgery was used to treat both lesional and nonlesional insular epilepsy, 53\% of patients achieved seizure freedom. ${ }^{11}$ This study reported Engel class I outcome in 6 of their 15 MRI-negative cases. In most of our cases, LITT was performed in the dominant hemisphere. No permanent deficits were noted after the procedures. Our 3 cingulate cases underwent LITT of the left cingulate cortex. Our combined insular and cingulate case also underwent left insular and cingulate LITT. To the best of our knowledge, there have been only case reports on nonlesional cingulate LITT. ${ }^{18,19}$ In our series, the 3 patients (cases 7-9) with only left cingulate seizure onset had favorable seizure outcome (Engel class I-III) following cingulate LITT. The patient (case 2) with both left cingulate and insular seizure onsets who underwent both left insular and cingulate LITT achieved Engel class IB outcome.

One of our patients who underwent insular LITT (case 4) experienced only a partial improvement in seizure outcome following insular LITT because of insufficient initial sEEG sampling. This patient underwent sEEG monitoring early in our sEEG experience, before we began sampling from the cingulate gyrus. Her seizure semiology consisted of whole-body stiffening followed by hypermotor activity including bicycling behavior and body rotation. With a limited sEEG array, her seizure onset was found to originate from the right insula. Following right-sided insular ablation, the patient's seizure semiology changed, with resolution of hypermotor activity, but whole-body stiffening continued. She subsequently underwent reevaluation with bilateral subdural grids and sEEG electrodes. Because monitoring provided poor seizure lateralization, she underwent a corpus callosotomy with her electrode array in place. Following callosotomy, her seizures arose from the left anterior cingulate. An open cingulate topectomy was performed, resulting in a transient expressive aphasia that persisted for 3 months. During her recovery she was able to text but could not speak. She ultimately recovered expressive language skills and achieved complete seizure freedom (Engel class IA).

The other patient (case 5), who achieved partial seizure control (Engel class III) after initial LITT in the left insular cortex, underwent additional sEEG monitoring that demonstrated epileptogenic activity in insular tissue adjacent to the initial ablation. That patient achieved complete seizure freedom (Engel class IA) after an additional LITT procedure ablating the epileptogenic insular tissue adjacent to prior ablations (Fig. 3). This experience supports the report by Mullatti et al. that the extent of complex ablations should be supported by neurophysiological data. ${ }^{11}$

One patient (case 7) experienced reduced but persistent seizure activity following cingulate LITT. Review of the postoperative MRI suggested that the initial LITT did not completely ablate the targeted cingulate tissue. Additional LITT was offered, but the patient declined additional surgery.

The rate of any perioperative intracranial hemorrhage associated with sEEG in our series was $22.2 \%$ ( 2 of 9), 
and the rate of symptomatic hemorrhage was $11.1 \%$ (1 of 9). The bleeding associated with the sEEG implantation in our cohort is similar to that reported in a recent analysis. ${ }^{20}$ Also, a large intraparenchymal hemorrhage occurred during LITT when multiple trajectories were used for an insular ablation (case 6, Supplementary Fig. S2). This hemorrhage required immediate cessation of LITT and emergency craniectomy with a large duraplasty. That patient recovered to preoperative neurological status with rehabilitation over a 12-month postoperative period and Engel class IA seizure outcome. It may be reasonable to consider emergency decompressive craniectomy with duraplasty when LITT-associated hemorrhages occur. Rates of perioperative hemorrhage following sEEG and LITT in nonlesional insular and cingulate epilepsy are not well described. In a large series of 549 patients in whom sEEG electrodes were implanted, 105 (19.1\%) experienced hemorrhage, with $16.9 \%$ asymptomatic and $2.2 \%$ being symptomatic. ${ }^{20}$ Reported rates of hemorrhage following LITT in the hippocampus and for hypothalamic hamartomas fall in the range of $7 \%-8 \% .^{21}$ It is possible that rates of hemorrhage from sEEG and/or LITT will be higher than in other structures, such as the hippocampus, due to the highly vasculature nature of the insula. The cause of hemorrhage in this case is unclear, and the cerebral angiogram was unrevealing. Large blood vessels are thought to have enough blood flow to effectively transfer heat and prevent rupture, and small vessels are likely to get ablated. However, there may be middle-sized vessels of unknown diameter that do not possess the heat sink protective properties of larger vessels, thereby leading to rupture and bleeding. ${ }^{11}$

Case series involving patients with insular or cingulate epilepsy surgery often describe transient neurological deficits including paresis, dysphasia, alteration of taste, smell, hearing, pain, and thermal perceptions. These transient symptoms can occur in up to $75 \%$ of cases..$^{22}$ In our series, 4 patients $(44.4 \%)$ developed neurological deficits following LITT and in 3 of 4 (33.3\% of all patients) these deficits were transient. Three patients who underwent insular LITT experienced transient expressive aphasia postprocedure. The patient in case 5 experienced a severe mixed expressive aphasia worse than receptive aphasia following her initial LITT procedure (Fig. 3). At her second LITT, she had a 48-hour period of marked word-finding difficulty during which she self-corrected with frustration. The patient in case 2 in our series underwent both insular and cingulate LITT based on the sEEG findings indicating seizure onset from both locations. In this patient, LITT on both insular and cingulate cortex was done on the same day and he developed a transient SMA syndrome.

\section{Interpretation}

The increased availability of sEEG has improved our understanding of cingulate and insular epilepsy, which has led to increased surgical treatment for DRE arising from insular and cingulate regions. This experience has revealed the complexity of clinical semiology for both insular and cingulate seizures. ${ }^{23}$ The semiology for patients with insular epilepsy in our cohort included activity arrest, staring, dystonic posturing, head drop, hypermotor activity, tingling sensation in the throat, and lip smacking. Cingulate semiologies in our series included contralateral dystonic posturing as well as hypermotor activity. These patterns of seizure activity may be caused by the spread of the seizure activity originating from the insula and cingulum.

Because insular seizure activity is known to spread rapidly between hemispheres, we implanted sEEG electrodes bilaterally in 5 of the 6 patients who underwent insular LITT. ${ }^{24}$ Previous series reported that open resection of the insula is associated with a risk of transient neurological comorbidities as high as $75 \% .^{22}$ In our LITT cohort, transient neurological deficits occurred in 4 of our 9 patients $(44 \%)$. Even though we performed the majority (5 of 6) of our ablations in the dominant hemisphere, there was no significant change in neuropsychological test scores following LITT.

\section{Limitations}

Ours is a small retrospective case series with insufficient case volume to allow statistical analysis. The cohort is heterogeneous both demographically and clinically. One-third of the patients underwent prior unsuccessful epilepsy surgery. All of our cases were nonlesional, and no insular or cingulate tissue specimens were obtained. We are therefore unable to determine the pathological cause of seizures in our series. A prior case series reported that 5 of 6 patients with insular epilepsy had focal cortical dysplasia. $^{25}$

In one of our cases, LITT was repeated in insular tissue immediately adjacent to the prior ablation cavity after the patient experienced recurrent seizure activity. In cases of DRE without a clear lesion and with limited sEEG monitoring volumes, it can be challenging to determine how much tissue to ablate with LITT. It also suggests that in some cases, a limited LITT of the insular region may not be adequate to achieve seizure freedom.

\section{Conclusions}

The nonlesional insular and cingulate DRE cases can be effectively localized with SEEG and treated with LITT. The complication rates associated with LITT in these locations are comparable to those following open insular resection. The rates of hemorrhage may be higher than reported in open insular resection and LITT in other locations in the brain, which may have to be considered while making therapeutic decisions. An insular or cingulate seizure onset zone in the dominant hemisphere is not a contraindication to LITT. We conclude that sEEG followed by LITT is a reasonable treatment option for DRE originating from these difficult-to-access regions of the cerebral cortex, particularly for patients with nonlesional epilepsy.

\section{References}

1. Centers for Disease Control and Prevention (CDC). Epilepsy in adults and access to care-United States, 2010. MMWR Morb Mortal Wkly Rep. 2012;61(45):909-913.

2. Del Felice A, Beghi E, Boero G, et al. Early versus late remission in a cohort of patients with newly diagnosed epilepsy. Epilepsia. 2010;51(1):37-42.

3. Englot DJ, Rolston JD, Wang DD, et al. Seizure outcomes after temporal lobectomy in pediatric patients. J Neurosurg Pediatr. 2013;12(2):134-141. 
4. Ansari SF, Tubbs RS, Terry CL, Cohen-Gadol AA. Surgery for extratemporal nonlesional epilepsy in adults: an outcome meta-analysis. Acta Neurochir (Wien). 2010;152(8):1299_ 1305.

5. Jobst BC, Cascino GD. Resective epilepsy surgery for drug-resistant focal epilepsy: a review. JAMA. 2015;313(3): 285-293.

6. Desai A, Bekelis K, Darcey TM, Roberts DW. Surgical techniques for investigating the role of the insula in epilepsy: a review. Neurosurg Focus. 2012;32(3):E6.

7. Afif A, Becq G, Mertens P. Definition of a stereotactic 3-dimensional magnetic resonance imaging template of the human insula. Neurosurgery. 2013;72(1)(Suppl Operative): 35-46.

8. Surbeck W, Bouthillier A, Weil AG, et al. The combination of subdural and depth electrodes for intracranial EEG investigation of suspected insular (perisylvian) epilepsy. Epilepsia. 2011;52(3):458-466.

9. Guénot M, Isnard J, Ryvlin P, et al. SEEG-guided RF thermocoagulation of epileptic foci: feasibility, safety, and preliminary results. Epilepsia. 2004;45(11):1368-1374.

10. Cardinale F, Cossu M, Castana L, et al. Stereoelectroencephalography: surgical methodology, safety, and stereotactic application accuracy in 500 procedures. Neurosurgery. 2013; 72(3):353-366.

11. Mullatti N, Landre E, Mellerio C, et al. Stereotactic thermocoagulation for insular epilepsy: lessons from successes and failures. Epilepsia. 2019;60(8):1565-1579.

12. Alkawadri R, So NK, Van Ness PC, Alexopoulos AV. Cingulate epilepsy: report of 3 electroclinical subtypes with surgical outcomes. JAMA Neurol. 2013;70(8):995-1002.

13. Hale AT, Sen S, Haider AS, et al. Open resection versus laser interstitial thermal therapy for the treatment of pediatric insular epilepsy. Neurosurgery. 2019;85(4):E730-E736.

14. Alomar S, Mullin JP, Smithason S, Gonzalez-Martinez J. Indications, technique, and safety profile of insular stereoelectroencephalography electrode implantation in medically intractable epilepsy. J Neurosurg. 2018;128(4):1147-1157.

15. Obaid S, Zerouali Y, Nguyen DK. Insular epilepsy: semiology and noninvasive investigations. J Clin Neurophysiol. 2017;34(4):315-323.

16. Freri E, Matricardi S, Gozzo F, et al. Perisylvian, including insular, childhood epilepsy: presurgical workup and surgical outcome. Epilepsia. 2017;58(8):1360-1369.

17. Weil AG, Le NM, Jayakar P, et al. Medically resistant pediatric insular-opercular/perisylvian epilepsy. Part 2: outcome following resective surgery. J Neurosurg Pediatr. 2016;18(5): 523-535.

18. Upadhyayula PS, Rennert RC, Hoshide R, et al. Laser ablation of a nonlesional cingulate gyrus epileptogenic zone using robotic-assisted stereotactic EEG localization: a case report. Stereotact Funct Neurosurg. 2019;97(1):10-17.

19. Marashly A, Loman MM, Lew SM. Stereotactic laser ablation for nonlesional cingulate epilepsy: case report. J Neurosurg Pediatr. 2018;22(5):481-488.
20. McGovern RA, Ruggieri P, Bulacio J, et al. Risk analysis of hemorrhage in stereo-electroencephalography procedures. Epilepsia. 2019;60(3):571-580.

21. Shukla ND, Ho AL, Pendharkar AV, et al. Laser interstitial thermal therapy for the treatment of epilepsy: evidence to date. Neuropsychiatr Dis Treat. 2017;13:2469-2475.

22. Bouthillier A, Nguyen DK. Epilepsy surgeries requiring an operculoinsular cortectomy: operative technique and results. Neurosurgery. 2017;81(4):602-612.

23. Nguyen DK, Nguyen DB, Malak R, et al. Revisiting the role of the insula in refractory partial epilepsy. Epilepsia. 2009; 50(3):510-520.

24. Afif A, Minotti L, Kahane P, Hoffmann D. Anatomofunctional organization of the insular cortex: a study using intracerebral electrical stimulation in epileptic patients. Epilepsia. 2010;51(11):2305-2315.

25. Gras-Combe G, Minotti L, Hoffmann D, et al. Surgery for nontumoral insular epilepsy explored by stereoelectroencephalography. Neurosurgery. 2016;79(4):578-588.

\section{Disclosures}

Dr. Baumgartner has served as a paid consultant for PMT Corp., iMRIs, Monteris, and Zimmer-Biomet.

\section{Author Contributions}

Conception and design: Gireesh, Baumgartner. Acquisition of data: Gireesh, Westerveld, Beegle, Baumgartner. Analysis and interpretation of data: Gireesh, Chen. Drafting the article: Gireesh, Chen. Critically revising the article: Lee, Skinner, Seo, Chen, Westerveld, Beegle, Castillo, Baumgartner. Reviewed submitted version of manuscript: Gireesh. Approved the final version of the manuscript on behalf of all authors: Gireesh. Statistical analysis: Gireesh. Study supervision: Gireesh, Baumgartner.

\section{Supplemental Information Online-Only Content}

Supplemental material is available with the online version of the article.

Supplementary Tables and Figures. https://thejns.org/doi/ suppl/10.3171/2020.7.JNS201912.

\section{Previous Presentations}

Presented as "Intracranial EEG and LASER Ablation in NonLesional Insular and Cingulate Epilepsy-Case Series" at the American Epilepsy Society conference in Baltimore, on December 7, 2019.

\section{Correspondence}

Elakkat D. Gireesh: Neuroscience Institute, AdventHealth, Orlando, FL. elakkat.gireesh.md@adventhealth.com. 